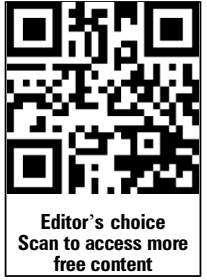

- Additional material is published online only. To view please visit the journal online (http://dx.doi.org/10.1136/ heartjnl-2014-306093).

${ }^{1}$ Department of Medical Sciences, Uppsala Clinical Research Center, Uppsala University, Uppsala, Sweden ${ }^{2}$ Department of Cardiology, Falun Hospital, Falun, Sweden ${ }^{3}$ Department of Cardiology, Lund University, Skane University Hospital, Lund, Sweden

${ }^{4}$ Department of Cardiology, Karolinska Institute, Karolinska University Hospital, Huddinge, Stockholm, Sweden

\section{Correspondence to} Dr Tomasz Baron, Department of Medical Sciences, Uppsala Clinical Research Center, Uppsala University, Dag Hammarskjölds väg 14B, 1 tr, Uppsala 752 37, Sweden; tomasz.baron@ucr.uu.se

Received 24 April 2014 Revised 11 September 2014 Accepted 22 September 2014 Published Online First 20 October 2014

\section{SLinked}

- http://dx.doi.org/10.1136/ heartjnl-2014-306865

\section{CrossMark}

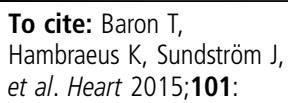

\title{
Type 2 myocardial infarction in clinical practice
}

\author{
Tomasz Baron, ${ }^{1}$ Kristina Hambraeus, ${ }^{2}$ Johan Sundström, ${ }^{1}$ David Erlinge, ${ }^{3}$ \\ Tomas Jernberg, ${ }^{4}$ Bertil Lindahl, ${ }^{1}$ TOTAL-AMI study group
}

\section{ABSTRACT \\ Objective We aimed to assess differences in incidence, clinical features, current treatment strategies and outcome in patients with type 2 vs. type 1 acute myocardial infarction (AMI).}

Methods and results All 20138 hospitalisations in Sweden with a diagnosis of AMI registered during 2011 in the Swedish Web-system for Enhancement and Development of Evidence-based care in Heart disease Evaluated According to Recommended Therapies were classified into types 1-5 in accordance with the universal definition of myocardial infarction (MI) from 2007. Type 1 AMI was present in $88.5 \%$ of the cases while $7.1 \%$ were classified as type 2 AMI. Higher age, female sex, comorbidities, impaired renal function, anaemia and smaller extent of myocardial necrosis characterised patients with type 2 AMI. While normal coronary arteries were more frequently seen $(42.4 \%$ vs. $7.4 \%)$, an invasive treatment was less common, and antiplatelet medications were less prescribed in patients with type 2 AMI compared with type 1 AMI. The group with type 2 AMI had significantly higher crude 1-year mortality compared with the group with type $1 \mathrm{AMI}$ (24.7\% vs. $13.5 \%, p<0.001)$. However, after adjustment, the HR for 1-year mortality in patients with type 2 AMl was $1.03(95 \% \mathrm{Cl} 0.86$ to 1.23$)$.

Conclusions In this real-life study, $7.1 \%$ of myocardial infarctions were classified as type 2 AMI. These patients were older, predominantly women and had more comorbidities. Invasive treatment strategies and cardioprotective medications were less used. Patients with type 2 AMI had higher crude mortality compared with type 1 patients with MI. However, after adjustment, the 1-year mortality was similar.

\section{INTRODUCTION}

In The Universal Definition of Myocardial Infarction, published in $2007,{ }^{1}$ five different clinical types of acute myocardial infarction (AMI) were introduced; the definitions of the five types have recently been updated in The Third Universal Definition of Myocardial Infarction. ${ }^{2}$ Type 1 AMI is caused by an acute atherothromboembolic coronary event. Type 2 AMI, also known as secondary $\mathrm{AMI}$, is a more heterogeneous entity, where a condition other than coronary artery disease (CAD) contributes to an acute imbalance between oxygen supply and demand. Type 2 AMI might occur in a patient with or without significant CAD. Type 3 is reserved for the rare cases where death occurs in patients with symptoms suggestive of myocardial ischaemia, and with presumed new ischaemic ECG changes before blood samples could be obtained or before cardiac biomarker could rise. Types 4 and 5 are iatrogenic infarctions associated with percutaneous coronary intervention and coronary artery bypass grafting, respectively.

In clinical practice, it may be difficult to distinguish type 2 AMI from type $1 \mathrm{AMI}$ and to distinguish type 2 AMI from other non-ischaemic conditions associated with myocardial damage and troponin elevation, such as myocarditis, septic shock or Takotsubo cardiomyopathy. ${ }^{3}$ Hence, large variations in the prevalence of type 2 AMI have been reported in the literature, ranging from $1.6 \%$ to $29.6 \%$. $^{4-7}$

By contrast with type 1 AMI, evidence-based treatment recommendations for type 2 AMI are lacking; and the knowledge about treatment in clinical practice and outcome is limited.

Therefore, the aim of this study was to compare the incidence, patient characteristics, pharmacological and invasive treatment, complications and 1-year mortality between patients with type 2 and type 1 AMI in a large contemporary cohort of patients with AMI included in a nationwide quality registry.

\section{METHODS}

\section{Study population}

Consecutive patients with AMI admitted to a cardiac or medical intensive care unit at all 73 hospitals in Sweden between 1 January and 31 December 2011 recorded in the Swedish Web-system for Enhancement and Development of Evidence-based care in Heart disease Evaluated According to Recommended Therapies (SWEDEHEART). The registry, which has a completeness of $79 \%$ of all AMIs diagnosed at Swedish hospitals according to the mandatory National Patient (diagnosis) Registry, ${ }^{8} 9$ contains data about baseline characteristics, ECG changes, biochemical markers, treatment and outcome (see (http://www. swedeheart.se) for details). Since 2010, classification of the AMI into types $1-5$ is included in the registry. The classification is done by the treating physician according to the universal definition.

To ensure the quality of the data entered into the database, a monitor visits approximately 10-20 hospitals (out of a total of 73 hospitals in Sweden) each year. During 2011, 13 units were monitored. There was a $96.1 \%$ agreement between data in the register and the information in the patients' records in 30-40 randomly chosen patients for each hospital (http://www.swedeheart.se).

According to Swedish law, all patients must be informed about their participation in the registry and the right to get their data erased from the registry on request. During 2011, no patient 
requested to get the data erased. The study was approved by the Regional Ethical Review Board at Uppsala University.

\section{Follow-up and outcome}

The prespecified primary outcome was 1-year all-cause mortality, and the follow-up data regarding mortality were available by merging the Swedish National Population Registry data until 31 December 2012 with SWEDEHEART on the basis of the personal identification number that all Swedish citizens have. Patients were followed-up from admission date to occurrence of primary outcome or until day 365 . No patient was lost to follow-up.

\section{Statistical methods}

Patients with type 1 and type 2 AMI were compared with each other. Categorical variables are presented as frequency values and compared by $\chi^{2}$ tests. Continuous variables are presented as mean \pm SD or as medians and IQRs. Differences in continuous variables were evaluated using independent samples $t$ tests and Mann-Whitney tests, as appropriate.

For analysis of mortality outcomes, only the first hospitalisation for each patient during 2011 was used, while all the registered hospitalisations were used to study clinical characteristics.

The univariate and multivariate associations between AMI type and invasive procedures and medical treatment during hospitalisation, in-hospital complications and medical treatment on discharge were assessed with different logistic regression models.

In the models, adjustment was made for the following variables in several different combinations: age, sex, smoking, medical history (hypertension, diabetes, congestive heart failure, previous myocardial infarction, stroke), treatment (revascularisation during hospitalisation, medications at discharge, e.g., ACE inhibitors, angiotensin receptor blockers, warfarin, aspirin, other antiplatelet drugs, $\beta$-blockers, statins) and treating centre. The variables were chosen based on clinical considerations and confounders known from risk-stratification models. Results from the logistic regression models were presented as ORs, with 95\% CIs.

Cumulative hazard curves were constructed using the KaplanMeier method and the log-rank test was used to compare survival distribution between type 1 and type 2 AMI during 1-year follow-up. In the multivariable analysis comparing 1 -year mortality, we used a Weibull regression model. ${ }^{10}$ Since treatments strategies might vary by reporting centre, we performed frailty analysis using treating hospital as random effect.

For all analyses, two-sided $\mathrm{p}$ values $<0.05$ were defined as significant. The $\mathrm{p}$ values from these tests should be interpreted descriptively due to the multiple testing. Statistical analyses were performed using IBM SPSS V.21.0 (SPSS, IBM Corporation, Armonk, New York, USA) and R V.3.1.1 (R Foundation for Statistical Computing, Vienna, Austria).

The authors had full access to, and take full responsibility for, the integrity of the data. All authors have read and agreed to the manuscript as written.

\section{RESULTS}

A total of 17951 patients, of whom 791 (4.4\%) had more than one hospitalisation with a discharge diagnosis of AMI, were reported to the SWEDEHEART registry by the 73 participating hospitals between January 1 and 31 December 2011, giving a total of 20138 hospitalisations with a diagnosis of AMI. The AMIs were classified into the 5 clinical types, as shown in figure 1 . The proportion of type 2 AMI varied considerably between the reporting sites with a median of $6.7 \%$ (10th-90th percentile: $0.2 \%-13.0 \%$ ). The most common discharge diagnoses in addition to AMI in the patients with type 2 AMI were tachyarrhythmia, heart failure, infection, anaemia, bleeding and renal impairment (table 1).

\section{Clinical characteristics, coronary angiography and laboratory findings}

Patients with type 2 AMI were older, more often women and had more cardiovascular risk factors and comorbidities, particularly heart failure and atrial fibrillation, compared with patients with type 1 (table 2).

Furthermore, patients with type 2 AMI were more often on treatment with $\beta$-blockers, renin-angiotensin-aldosterone system

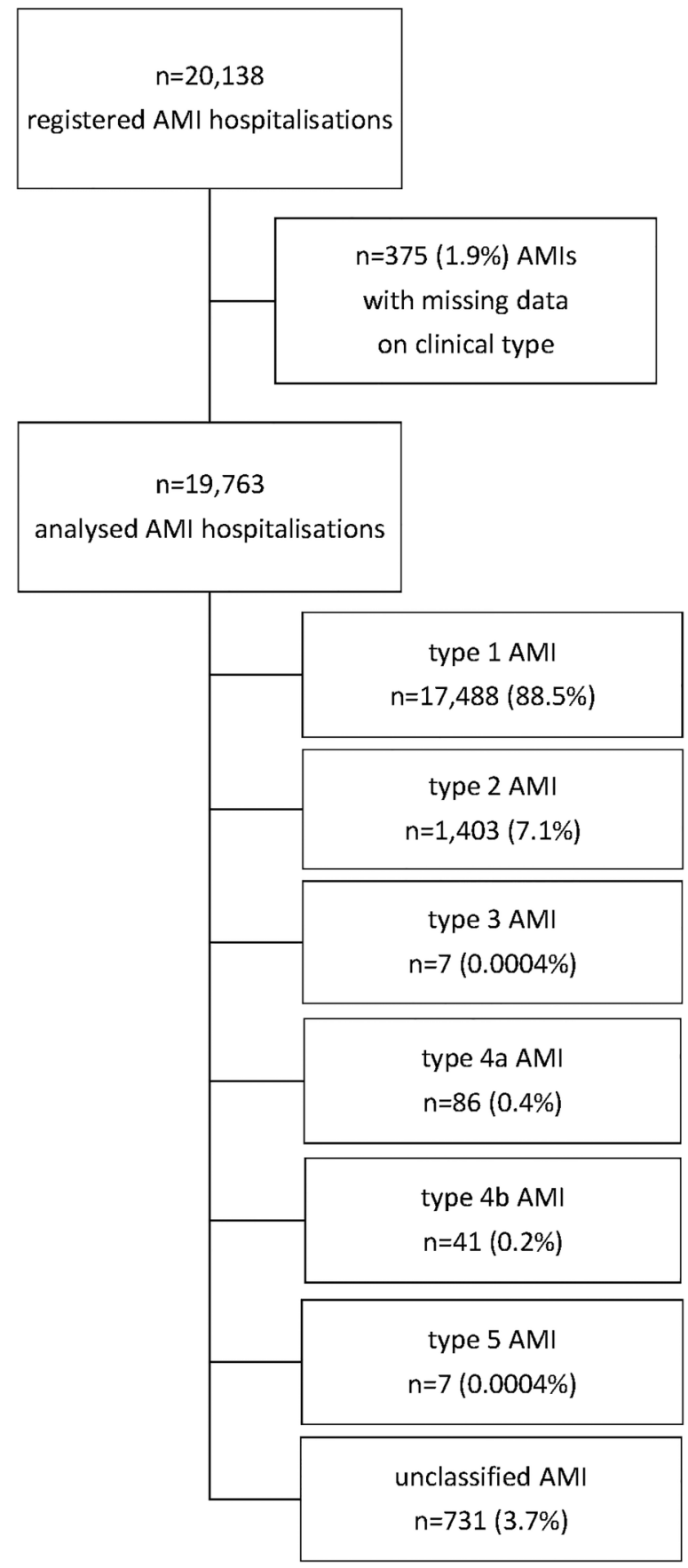

Figure 1 Classification of clinical acute myocardial infarction (AMI) types. 
Table 1 The most common discharge diagnoses (other than AMI) in patients with type $2 \mathrm{AMI}$, most likely representing the triggering mechanisms

\begin{tabular}{ll}
\hline Diagnosis* & Number of diagnoses \\
\hline Tachyarrhythmia & 331 \\
Heart failure & 260 \\
Infection & 246 \\
Anaemia/bleeding & 186 \\
Renal insufficiency & 82 \\
COPD/asthma exacerbation & 78 \\
Hypertensive crisis & 30 \\
Stroke/TIA & 24 \\
Respiratory insufficiency & 19 \\
\hline
\end{tabular}

* More than one diagnosis per patient/hospitalisation is possible.

AMI, acute myocardial infarction; COPD, chronic obstructive pulmonary disease;

TIA, transient ischaemic attack.

blockade, statins, antiplatelet drugs, anticoagulants, diuretics and digoxin on admission (table 1). Of the patients who underwent invasive coronary angiography during hospitalisation, normal coronary arteries or non-obstructive CAD ( $<50 \%$ stenosis) were significantly more common in type 2 vs. type 1 AMI (42.4\% vs. $7.4 \%, \mathrm{p}<0.001)$. Patients with type 2 AMI showed higher values of creatinine, C-reactive protein and plasma glucose, while their haemoglobin and cholesterol levels were lower on admission (table 3).

Moreover, their peak cardiac troponin (cTn) concentrations were lower compared with patients with type $1 \mathrm{AMI}$, independent of the type of biomarker used. The types of cTn assays used

Table 2 Demographics, medical history and medications on admission

\begin{tabular}{lclr}
\hline & $\begin{array}{l}\text { Type 1 AMI } \\
\mathbf{n}=17 \text { 488 }\end{array}$ & $\begin{array}{l}\text { Type 2 AMI } \\
\mathbf{n = 1 4 0 3}\end{array}$ & p Value \\
\hline Demographics & & & \\
Age, year $( \pm$ SD) & $71.1( \pm 12.5)$ & $75.9( \pm 11.4)$ & $<0.001$ \\
Female sex, \% & 34.2 & 46.8 & $<0.001$ \\
Cardiovascular risk factors and medical history & & \\
Smoking, \% & 58.6 & 54.5 & 0.006 \\
Hypertension, \% & 50.7 & 54.2 & 0.011 \\
Diabetes, \% & 22.2 & 26.8 & $<0.001$ \\
History of PCI, \% & 17.6 & 17.4 & 0.836 \\
History of CABG, \% & 10.0 & 14.7 & $<0.001$ \\
History of AMI, \% & 30.4 & 40.1 & $<0.001$ \\
History of CHF, \% & 10.6 & 20.5 & $<0.001$ \\
History of stroke, \% & 9.2 & 13.9 & $<0.001$ \\
Medications & & & \\
ACE inhibitors, \% & 25.3 & 32.9 & 0.009 \\
ARB, \% & 15.0 & 17.6 & $<0.001$ \\
B-blockers, \% & 41.5 & 55.9 & $<0.001$ \\
Digitalis, \% & 2.1 & 3.8 & $<0.001$ \\
Aspirin, \% & 41.5 & 53.2 & $<0.001$ \\
Other antiplatelets, \% & 9.7 & 12.9 & $<0.001$ \\
Oral anticoagulants, \% & 5.3 & 10.2 & $<0.001$ \\
Statins, \% & 34.3 & 42.9 & $<0.001$ \\
Diuretics, \% & 26.5 & 41.6 & $<0.001$ \\
\hline ACE angions & &
\end{tabular}

$A C E$, angiotensin converting enzyme; $A M I$, acute myocardial infarction; $A R B$, angiotensin receptor blocker; $C A B G$, coronary artery bypass grafting; $C H F$, congestive heart failure; $\mathrm{PCl}$, percutaneous coronary intervention. by the treating centres are shown in online supplementary table S1. In patients with type 2 AMI, the admission ECG showed ST-segment depression, left bundle branch block (LBBB) or no ischaemic ST-T changes more often than in patients with type 1 AMI (table 2). By contrast, ST-segment elevation was less common, but not totally absent, in patients with type 2 AMI.

\section{In-hospital treatment and medication on discharge}

Patients with type 2 AMI underwent invasive coronary angiography less frequently and were less often revascularised compared with patients with type $1 \mathrm{AMI}$, the differences remained highly significant also after adjustment for age, sex and comorbidities (table 4).

During hospitalisation, the use of intravenous diuretics was more frequent in type $2 \mathrm{AMI}$ as compared with type $1 \mathrm{AMI}$, while use of intravenous/subcutaneous anticoagulants did not differ significantly between the groups (table 4).

On discharge, cardioprotective medications such as $\beta$-blockers and statins were less often prescribed to type 2 AMI patients. Antiplatelet drugs were also less often prescribed, while anticoagulants were more often prescribed to patients with type 2 AMI compared with type 1 (table 5).

These differences remained highly significant also after adjustment.

\section{In-hospital complications}

The incidence of in-hospital bleedings $(5.5 \%$ vs. $1.0 \%$, $\mathrm{p}<0.001)$, as well as onset of atrial fibrillation during hospitalisation $(6.9 \%$ vs. $3.9 \%, \mathrm{p}<0.001)$ were higher in type 2 versus type 1 AMI. After adjustment for age, sex and comorbidities, the resulting ORs were 1.47 (95\% CI 1.29 to 1.68) and 1.43 (95\% CI 1.19 to 1.73 ), respectively. The incidence of in-hospital cardiac arrest, cardiogenic shock and high-grade AV block were comparable $(2.0 \%$ vs. $3.2 \%, 1.6 \%$ vs. $2.1 \%, 1.4 \%$ vs. $1.5 \%$, respectively, $\mathrm{p}=\mathrm{ns}$ ) in both groups.

\section{Mortality}

The crude 1-year mortality was higher in type 2 AMI than in type 1 AMI, $24.7 \%$ vs. $13.5 \%$ ( $\mathrm{p}<0.001)$. The event curves separated early and continued to separate throughout the follow-up period (figure 2). In univariate analysis, the HR for 1-year mortality was 1.86 (95\% CI 1.66 to 2.08$)$ for type 2 as compared with type 1 AMI. However, after adjustment for background characteristics, treatments and clustering by treating hospitals, the difference in 1-year mortality was attenuated and did not reach statistical significance; HR 1.03 (95\% CI 0.86 to 1.23 ).

\section{DISCUSSION}

In this large real-life register study including 20138 AMI hospitalisations from all Swedish hospitals, $7.1 \%$ of the cases were diagnosed as type 2 AMI. The patients with type 2 AMI were older and had more comorbidities compared with patients with type 1 AMI. They underwent invasive diagnostic and/or revascularisation procedures, and were prescribed standard secondary preventive treatment less often. Patients with type 2 AMI showed worse outcome compared with patients with type 1 AMI; however, after adjustment for background factors and treatments, the mortality was similar.

In the few previous studies describing the incidence of type 2 AMI, the incidence varies from $1.6 \%$ to $29.6 \%,{ }^{4-7}$ which corresponds to the considerable variation in reported incidences between the hospitals in our study. The variation most probably reflects an uncertainty in the classification of the AMIs into the different subtypes and underscores the lack of clear and 
Table 3 Symptoms, clinical signs, laboratory results and ECG-findings on admission

\begin{tabular}{|c|c|c|c|}
\hline & $\begin{array}{l}\text { Type } 1 \text { AMI } \\
\mathrm{n}=17488\end{array}$ & $\begin{array}{l}\text { Type } 2 \text { AMI } \\
n=1403\end{array}$ & $\mathrm{p}$ Value \\
\hline \multicolumn{4}{|l|}{ Main symptom } \\
\hline Chest pain, \% & 84.8 & 62.0 & $<0.001$ \\
\hline Dyspnoea, \% & 7.0 & 19.2 & $<0.001$ \\
\hline Other symptoms, \% & 8.2 & 18.8 & $<0.001$ \\
\hline \multicolumn{4}{|l|}{ Clinical status } \\
\hline Heart rate, bpm $( \pm S D)$ & $81.3( \pm 22.2)$ & $97.8( \pm 30.5)$ & $<0.001$ \\
\hline Heart rate $>150 \mathrm{bmp}, \%^{*}$ & 0.8 & 5.2 & $<0.001$ \\
\hline Systolic BP, mm Hg ( \pm SD) & $147.6( \pm 29.6)$ & $140.4( \pm 31.2)$ & $<0.001$ \\
\hline Systolic BP $<90 \mathrm{~mm} \mathrm{Hg}$ \%* & 1.9 & 2.6 & 0.051 \\
\hline Systolic BP >160 mm Hg, \%* & 30.0 & 22.5 & $<0.001$ \\
\hline Diastolic BP, mm Hg ( \pm SD) & $84.1( \pm 17.5)$ & $79.3( \pm 18.0)$ & $<0.001$ \\
\hline Pulmonary rales, \% & 11.3 & 22.1 & $<0.001$ \\
\hline \multicolumn{4}{|l|}{ Laboratory results, median (IQR) } \\
\hline Hs-Troponin T, ng/L (max) & $\begin{array}{l}351.5(84.0-1432.0) \\
(n=11182)\end{array}$ & $\begin{array}{l}247.0(89.4-721.3) \\
(n=902)\end{array}$ & $<0.001$ \\
\hline Troponin I, $\mu g / L$ (max) & $\begin{array}{l}3.1(0.59-16.0) \\
(n=5079)\end{array}$ & $\begin{array}{l}1.5(0.41-6.30) \\
(n=469)\end{array}$ & $<0.001$ \\
\hline Total cholesterol, mmol/L & $4.9(4.1-5.8)$ & $4.4(3.6-5.3)$ & $<0.001$ \\
\hline Triglycerides, mmol/L & $1.32(1.0-1.9)$ & $1.2(0.9-1.7)$ & $<0.001$ \\
\hline Glucose, $\mathrm{mmol} / \mathrm{L}$ & $7.0(6.0-9.1)$ & $7.6(6.1-10.5)$ & $<0.001$ \\
\hline Creatinine, $\mathrm{mmol} / \mathrm{L}$ & $84.0(70.0-104.0)$ & $92.0(73.0-125.0)$ & $<0.001$ \\
\hline eGFR, $\mathrm{mL} / \mathrm{min}$ & $76.7(58.6-93.5)$ & $64.1(45.5-83.1)$ & $<0.001$ \\
\hline eGFR<60 mL/min, \%* & 26.7 & 44.5 & $<0.001$ \\
\hline CRP, mg/L & $5.0(2.6-14.0)$ & $9.0(4.2-46.0)$ & $<0.001$ \\
\hline $\mathrm{Hb}, \mathrm{mg} / \mathrm{L}$ & $138.0(126.0-149.0)$ & $127.0(111-0-141.0)$ & $<0.001$ \\
\hline $\mathrm{Hb}<89 \mathrm{mg} / \mathrm{L}(\mathrm{M})$ or & & & $<0.001$ \\
\hline$<81 \mathrm{mg} / \mathrm{L}(\mathrm{F}), \% *$ & 0.3 & 4.1 & \\
\hline $\mathrm{Hb} \leq 109 \mathrm{mg} / \mathrm{L}, \% \dagger$ & 6.3 & 22.7 & $<0.001$ \\
\hline \multicolumn{4}{|l|}{ ECG-findings } \\
\hline ST-segment elevation, \% & 31.7 & 9.7 & $<0.001$ \\
\hline ST-segment depression, \% & 22.7 & 31.8 & $<0.001$ \\
\hline LBBB, \% & 6.3 & 11.6 & $<0.001$ \\
\hline No ischaemic changes, \% & 22.2 & 25.9 & $<0.001$ \\
\hline Sinus rhythm, \% & 86.6 & 67.0 & $<0.001$ \\
\hline Atrial fibrillation/flutter, \% & 10.4 & 28.1 & $<0.001$ \\
\hline Other rhythm, \% & 2.7 & 4.1 & $<0.001$ \\
\hline
\end{tabular}

unambiguous diagnostic criteria, where it is difficult to differentiate type 1 from type 2 AMI and also type 2 AMI from myocardial injury of multifactorial genesis. ${ }^{1-3}$
In accordance with previous, much smaller studies, the patients with type 2 AMI compared with patients with type 1 AMI in the present study were older, ${ }^{7}$ more often women, ${ }^{6}$ had

Table 4 Invasive procedures and medical treatment during hospitalisation

\begin{tabular}{|c|c|c|c|c|c|}
\hline & $\begin{array}{l}\text { Type } 1 \text { AMI } \\
\mathrm{n}=17488\end{array}$ & $\begin{array}{l}\text { Type } 2 \text { AMI } \\
n=1403\end{array}$ & p Value & OR $(95 \% \mathrm{Cl})$ & p Value* \\
\hline \multicolumn{6}{|l|}{ Invasive procedures } \\
\hline Coronary angiography, \% & 77.3 & 35.9 & $<0.001$ & $0.16(0.14$ to 0.20$)$ & $<0.001$ \\
\hline $\mathrm{PCl}, \%$ & 60.6 & 12.5 & $<0.001$ & $0.10(0.08$ to 0.13$)$ & $<0.001$ \\
\hline CABG, \% & 5.2 & 1.1 & $<0.001$ & $0.23(0.13$ to 0.41$)$ & $<0.001$ \\
\hline \multicolumn{6}{|l|}{ Medical treatments } \\
\hline Anticoagulants intravenous/subcutaneous, \% & 66.6 & 64.6 & 0.194 & $1.11(0.97$ to 1.28$)$ & 0.136 \\
\hline Diuretics intravenous, \% & 19.1 & 33.2 & $<0.001$ & 1.74 (1.48 to 2.04$)$ & $<0.001$ \\
\hline
\end{tabular}

${ }^{*}$ Adjusted for age, sex and comorbidities.

$\mathrm{AMI}$, acute myocardial infarction; $\mathrm{CABG}$, coronary artery bypass grafting; $\mathrm{PCI}$, percutaneous coronary intervention. 
Table 5 Medical treatment on discharge

\begin{tabular}{|c|c|c|c|c|c|}
\hline & $\begin{array}{l}\text { Type } 1 \text { AMI } \\
n=17488\end{array}$ & $\begin{array}{l}\text { Type } 2 \text { AMI } \\
n=1403\end{array}$ & p Value & $\begin{array}{l}\text { OR }(95 \% \mathrm{CI}) \\
\text { Type } 2 \text { vs. type } 1 \mathrm{AMI} \\
\text { (multiple adjusted)* }\end{array}$ & p Value* \\
\hline ACE inhibitors, \% & 60.6 & 48.8 & $<0.001$ & 0.89 (0.76 to 1.03$)$ & 0.102 \\
\hline$A R B, \%$ & 16.2 & 18.0 & 0.083 & 1.00 (0.84 to 1.18$)$ & 0.990 \\
\hline$\beta$-blockers, \% & 87.5 & 81.7 & $<0.001$ & 0.73 (0.59 to 0.89$)$ & 0.002 \\
\hline Aspirin, $\%$ & 92.6 & 74.2 & $<0.001$ & $0.37(0.31$ to 0.45$)$ & $<0.001$ \\
\hline Other antiplatelets, \% & 81.4 & 46.7 & $<0.001$ & 0.36 (0.30 to 0.42$)$ & $<0.001$ \\
\hline Double antiplatelet treatment, \% & 75.9 & 40.2 & $<0.001$ & $0.38(0.33$ to 0.45$)$ & $<0.001$ \\
\hline Oral anticoagulants, $\%$ & 7.4 & 15.6 & $<0.001$ & 1.30 (1.06 to 1.60$)$ & 0.011 \\
\hline Digoxin, \% & 2.3 & 5.8 & $<0.001$ & $1.14(0.81$ to 1.62$)$ & 0.453 \\
\hline Statins, \% & 86.0 & 66.0 & $<0.001$ & $0.56(0.47$ to 0.68$)$ & $<0.001$ \\
\hline Diuretics, \% & 32.4 & 50.3 & $<0.001$ & 1.26 (1.08 to 1.47$)$ & 0.003 \\
\hline
\end{tabular}

more cardiovascular risk factors such as diabetes, ${ }^{6}$ more frequently had a history of previous cardiovascular events, ${ }^{6}$ anaemia, ${ }^{67}$ renal dysfunction, ${ }^{5} 611$ signs of infection/inflammation $^{6}$ and more often had atrial fibrillation and/or signs of acute heart failure at presentation. ${ }^{6} 7$ Higher prevalence of chronic obstructive pulmonary disease (COPD) and peripheral arterial disease in type 2 AMI have also been described, ${ }^{5}{ }^{6}$ which was not possible to address in our study.

As previously noted, ${ }^{6}$ ST segment elevation is rarely observed in type 2 AMI, but not totally absent. By contrast, and not previously described, occurrence of LBBB and absence of ischaemic changes in the ECG, respectively, were more common in type 2 AMI than in type $1 \mathrm{AMI}$ in the present study.

In line with previous experiences, we found that type 2 AMI was associated with substantially lower cTn levels than type 1 AMI, reflecting a smaller extent of myocardial damage in type $2 \mathrm{AMI}^{5}$

In the present study, coronary angiography was performed in $36 \%$ of the cases with type 2 AMI during the hospital stay, less than half of that in patients with type 1 AMI (77\%), but more

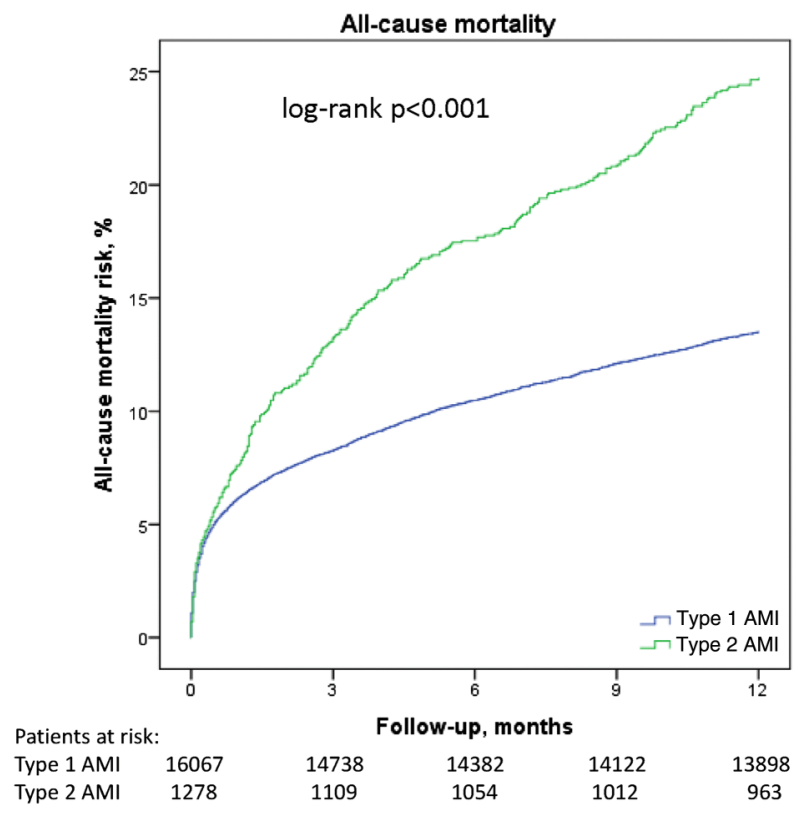

Figure 2 Crude cumulative risk of death (Kaplan-Meier) in patients with type 1 and type 2 acute myocardial infarction (AMI). often than the $22 \%$ in the study by Saaby et al. ${ }^{6}$ While normal coronary arteries or non-obstructive atheromatosis were more often seen, the subsequent revascularisations were significantly less common in type 2 AMI compared with type 1 AMI in the present study. These findings are in line with the study of Saaby et $a l^{6}$ showing that $48 \%$ of the patients with type 2 AMI who had coronary angiography performed $(n=31)$ had normal coronary arteries, and with another small study showing nonobstructive stenosis or normal coronary arteries in one-third of the patients with type 2 AMI as compared with only $10 \%$ of patients with type 1 AMI. ${ }^{11}$ On the other hand, type 2 AMI patients with significant stenosis appear to more often present with 3 -vessel disease $(32.3 \%$ vs. $26.6 \%, p<0.001)$ as compared with patients with type 1 non-ST elevation myocardial infarction. ${ }^{11}$ Taken together, these results confirm the heterogeneity regarding CAD among patients with type 2 AMI.

Data on how patients with type 2 AMI are treated in clinical practice are limited. In our study, patients with type 2 AMI less often received secondary preventive treatment such as $\beta$-blockers, statins and antiplatelets, while they more often received anticoagulants and diuretics probably reflecting the higher prevalence of atrial fibrillation and heart failure in this group. Contrary to our observations, Javed et $a l^{5}$ reported no difference in prescribed medication between type 1 and 2 AMI groups. The different findings in the two studies probably reflect the lack of evidencebased treatment recommendations and consensus among clinicians as to how to treat patients with type 2 AMI.

Consistent with the higher incidence of comorbidities, such as renal impairment and heart failure, patients with type 2 AMI had a higher rate of in-hospital bleedings and new onsets of atrial fibrillation compared with patients with type 1 AMI.

Not surprisingly, given the higher age and more frequent comorbidities in patients with type 2 AMI, a higher crude mortality was observed during 1-year follow-up in patients with type 2 vs. type 1 AMI in the present study. By contrast, no difference in crude in-hospital mortality was shown between patients with type 1 and type 2 AMI in a previous small study. ${ }^{5}$ However, after adjustment for background characteristics, treatments and reporting centre, the patients with type 2 AMI in the present study showed similar 1-year mortality, indicating that the higher crude mortality in type 2 AMI is caused by factors other than the AMI itself. These findings are in accordance with the study of Gonzalez et al. ${ }^{7}$ The similar adjusted 1-year mortality in patients with type 2 and type $1 \mathrm{AMI}$, despite significantly 
larger infarction by cTn levels in the latter ones, may be explained by a potentially higher incidence in patients with type 2 AMI of other important comorbidities (e.g., COPD and cancer) not reported in our registry, and thus not possible to adjust for in the analysis.

\section{LIMITATIONS}

There are some limitations to the present study that need to be considered. First, data from observational registries have lower quality and there are more often missing data as compared with randomised clinical trials. However, the incidence of missing data was quite low, $<1 \%$ for almost all variables in our study and the correctness of data entered in the SWEDEHEART registry have been shown to be quite good. ${ }^{8}$

Second, the classification of the AMI was done locally by the treating physician at each participating hospital, and the exact triggering factor of type 2 AMI was not ascertained.

Third, the lower rate of coronary angiography in type 2 AMI may, in part, reflect verification bias of an unexpected finding of culprit lesion, which can lead to reclassification to type 1 AMI.

\section{Key messages}

\section{What is already known on this subject?}

Type 2 myocardial infarction is caused by myocardial blood flow supply/demand imbalance leading to ischaemia and eventually, myocardial necrosis. The imbalance is triggered by other conditions than a primary atherothromboembolic coronary event (type 1 acute myocardial infarction (AMI)), e.g., anaemia or tachyarrhythmia, and may occur in patients with or without significant coronary artery disease. The clinical characteristics, treatment and outcome of patients with type $2 \mathrm{AMI}$ in clinical routine are not well studied.

\section{What might this study add?}

Type 2 AMI represented about 7\% of all myocardial infarctions in this large, real-life cohort of AMI patients. Patients with type 2 AMI were older, had more comorbidities and were more often treated conservatively compared with patients with type 1 AMI. Despite a higher crude mortality in patients with type $2 \mathrm{AMI}$, the adjusted 1-year mortality was similar in patients with type 2 and type 1 AMI.

\section{How might this impact on clinical practice?}

Patients with type $2 \mathrm{AMI}$ constitute a heterogeneous population with much comorbidity and adverse prognosis. Unfortunately, there is a lack of evidence-based treatment recommendations for type 2 AMI. However, it is reasonable to suggest that the initiating condition must be sought and aggressively treated if possible. Furthermore, whether the patient has significant coronary artery disease or not should be elucidated.
Fourth, compared with the mandatory National Patient Registry containing all discharge diagnoses from hospitals in Sweden, the coverage of the SWEDEHEART registry for hospitalisations with a diagnosis of AMI was $79 \%$ in $2011 .^{9}$ As the patients with type 2 AMI were older and had more comorbidities, they might more likely have been treated in clinical departments other than cardiac care units and, therefore, not registered in SWEDEHEART. Thus, the true incidence of type 2 AMI might be underestimated in the present study.

Acknowledgements The author would like to thank Nermin Hadziosmanovic, biostatistician at Uppsala Clinical Research Center for his contribution to this article.

Contributors All authors contributed to the study design and interpretation of results, and reviewed and approved the manuscript prior to submission. TB wrote the first manuscript draft and performed all analyses. BL chaired the TOTAL-AMI steering group and made the first critical revision of the manuscript. $\mathrm{KH}, \mathrm{DE}$ and TJ chaired working groups within TOTAL-AMI.

Funding This work was supported by a grant from the Swedish Foundation for Strategic Research. The SWEDEHEART registry is supported by the Swedish Society of Cardiology, the Swedish Society of Thoracic Radiology, the Swedish Society of Thoracic Surgery and the Swedish Heart Association. The registry is financed by the government and the Swedish Association of Local Authorities and Regions (SALAR).

Competing interests None.

Ethics approval Regional Ethical Review Board at Uppsala University, Sweden. Provenance and peer review Not commissioned; externally peer reviewed.

\section{REFERENCES}

1 Thygesen K, Alpert JS, White HD.; Joint ESC/ACCF/AHA/WHF Task Force for the Redefinition of Myocardial Infarction. Universal definition of myocardial infarction. Eur Heart J 2007;28:2525-38.

2 Thygesen K, Alpert JS, Jaffe AS, et al.; JointESC/ACCF/AHA/WHF Task Force for Universal Definition of Myocardial Infarction. Third universal definition of myocardial infarction. J Am Coll Cardiol 2012;60:1581-98.

3 Alpert JS, Thygesen KA, White HD, et al. Diagnostic and therapeutic implications of type 2 myocardial infarction: review and commentary. Am J Med 2014;127:105-8.

4 Melberg T, Burman R, Dickstein K. The impact of the 2007 ESC-ACC-AHA- WHF Universal definition on the incidence and classification of acute myocardial infarction: A retrospective cohort study. Int J Cardiol 2010;139:228-33.

5 Javed U, Aftab W, Ambrose JA, et al. Frequency of elevated troponin I and diagnosis of acute myocardial infarction. Am J Cardiol 2009;104:9-13.

6 Saaby L, Poulsen TS, Hosbond S, et al. Classification of myocardial infarction: frequency and features of type 2 myocardial infarction. Am J Med 2013;126:789-97.

7 Gonzalez MA, Eilen DJ, Marzouq RA, et al.; Multidisciplinary Atherosclerosis Prevention Program. The universal classification is an independent predictor of long-term outcomes in acute myocardial infarction. Cardiovasc Revasc Med 2011;12:35-40.

8 Jernberg T, Attebring MF, Hambraeus K, et al. The Swedish Web-system for enhancement and development of evidence-based care in heart disease evaluated according to recommended therapies (SWEDEHEART). Heart 2010;96:1617-21.

9 Harnek J, Nilsson J, Friberg O, et al. The 2011 outcome from the Swedish Health Care Registry on Heart Disease (SWEDEHEART). Scand Cardiovasc J 2013;47(Suppl 62):1-10.

10 Hosmer D, Lemeshow S, May S. Applied survival analysis: regression modeling of time to event data. New York, NY: A Wiley-Interscience Publication, John Wiley \& Sons Inc., 2011:231-306.

11 Ambrose JA, Loures-Vale A, Javed U, et al. Angiographic correlates in Type 1 and 2 MI by the universal definition. JACC Cardiovasc Imaging 2012;5:463-4. 